„DIE VAAM KÜRT 2014 ZUM ERSTEN MAL DIE MIKROBE DES JAHRES, MIT DER SIE BEDEUTENDE, FASZINIERENDE, OFT ÜBERSEHENE SEITEN DER MIKROORGANISMEN EIN STÜCK INS LICHT DER ÖFFENTLICHKEIT RÜCKEN WILL.“

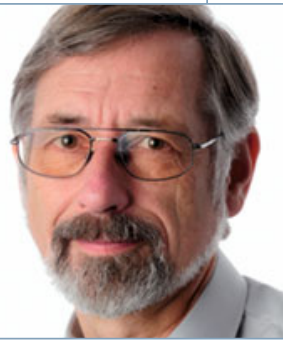

Harald Engelhardt

\title{
Mikroorganismen ins rechte Licht rücken
}

$10.1007 / \mathrm{s} 12268-014-0414-5$

(C) Springer-Verlag 2014

Wieso assoziieren die Menschen mit Mikroorganismen reflexartig Krankheit und Verdorbenes? Warum beachtet in unserer Gesellschaft kaum jemand die unglaubliche Bedeutung dieser Kleinstlebewesen, ohne die es viele Medikamente nicht gäbe, ohne die wir verhungerten, ohne die wir buchstäblich nicht leben könnten? Und weshalb werden wir nicht auch auf Mikroorganismen aufmerksam, wenn wir uns mit Neugier, Freude oder Sorge der Natur zuwenden?

Ein offensichtlicher Grund ist die vermeintliche Unsichtbarkeit der Mikroben; sie bleiben uns ohne Mikroskop als einzelne Wesen verborgen. Ein anderer liegt in ihrer Unscheinbarkeit; der Anblick einer Bakterienzelle macht gewöhnlich nicht viel her. Die tiefer liegende Ursache aber ist, dass wir nicht auf sie aufmerksam gemacht werden. Auch beeindruckende, bedrohte und versteckt lebende Tiere und Pflanzen nehmen wir erst wahr, wenn jemand unser Interesse anregt. Nur die Pathogenen unter den Bakterien erfreuen sich funktionierender Public Relations: Salmonellose, Borreliose, Legionellose, Meningitis - die Verursacher von Krankheiten prägen das öffentliche Bild von Mikroorganismen. Und das ist nicht unbedingt von Sympathie durchdrungen. Die UNESCO hat sogar - mit gutem Grund - einen Welttuberkulosetag eingerichtet, um diesem Übel den Kampf anzusagen. Und umfangreiche Forschungsaktivitäten widmen sich, hoffentlich mit Erfolg, der Eindämmung bakterieller Epidemien. Aber wir haben Bakterien mehr zu verdanken als Infektionen.

Die auf „Pest und Cholera“ fokussierte Wahrnehmung hat gewöhnlich nur einige Dutzend krankheitserregende Bakterienarten im Visier. Aber sie blendet Tausende bekannte und vermutlich mehrere Millionen noch zu entdeckende Arten aus. Wem ist bewusst, dass die Natur nicht ohne Mikroben auskäme, dass wesentliche Teile der globalen Stoffkreisläufe auf Bakterien und Archaeen beruhen, dass fast alle Organismen - vom Pantoffeltierchen bis zum Menschen - sich ohne Mikroorganismen nicht ernähren könnten? Und dass wohl alle Pflanzen, Tiere und Menschen ihr eigenes Mikrobiom besitzen, mit dem sie leben und von dem sie abhängen? Wer hat vor Augen, welche mikrobiellen Leistungen und biotechnologischen Dienste in unserem täglichen Leben eine unverzichtbare Rolle spielen?

Gewiss, die Wissen-Seiten der Zeitungen berichten über aktuelle Forschungsergebnisse, und die Forschungsinstitutionen verbreiten mitteilenswerte, oft spezielle Neuigkeiten von und mit Prokaryoten. Aber eingängige, verständliche und begründete Darstellungen der Vielfalt von Mikroorganismen und ihrer Bedeutung für Natur und Mensch findet man nicht leicht, trotz zahlreicher Beiträge im Internet. Auch im Biologieunterricht fristen Archaeen und Bakterien ein eher randständiges Dasein. Und so kann es geschehen, dass wir über Mikroben eher Diffuses aus der Probiotik-Werbung erfahren, vor allem aber Negatives, wenn die mikrobielle Diversität in der Natur außer Balance gerät oder pathogene Vertreter unsere Gesundheit bedrohen.

Die Mikrobiologie steht als Beispiel für andere biologische Bereiche, die ähnliche Schieflagen in der öffentlichen Aufmerksamkeit und Diskussion kennen. Das Manko selektiver Wahrnehmung oder Darstellung vorhandenen Wissens und erforschter Zusammenhänge mag man beklagen, aber wir sollten es nicht dabei belassen. Es ist auch die Aufgabe wissenschaftlicher Fachgesellschaften, ihre Erkenntnisse verständlich weiterzugeben. Öffentlichkeitsarbeit wird inzwischen von allen Universitäten und Forschungsinstituten als unverzichtbar erkannt und eingesetzt. Aber die ganze Breite biologischer Disziplinen können nur wissenschaftliche Gesellschaften angemessen repräsentieren. Und hier bieten sich zahlrei- che Möglichkeiten, man muss sie nur aufgreifen.

Die VAAM kürt 2014 zum ersten Mal die „Mikrobe des Jahres“, mit der sie bedeutende, faszinierende, oft übersehene Seiten der Mikroorganismen ein Stück ins Licht der Öffentlichkeit rücken will. Blättern Sie weiter und lassen Sie sich überraschen. Denn die in diesem Jahr ausgewählten Mikroben können Sie mit bloßem Auge sehen und mit ein wenig Glück auf einem Spaziergang selbst entdecken, vor allem nach einem Sommerregen. Interessiert? Mehr finden Sie auf S. 151 in dieser Ausgabe und unter www.mikrobe-des-jahres.de.

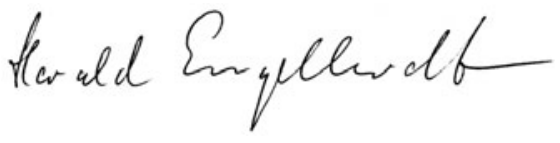

Harald Engelhardt

Korrespondenzadresse:

Dr. Harald Engelhardt

Max-Planck-Institut für Biochemie

Am Klopferspitz 18

D-82152 Martinsried

Tel.: 089-85782650

Fax: 089-8578-2641

engelhar@biochem.mpg.de 